\title{
Current surgical results of acute type A aortic dissection in Japan
}

\author{
Yutaka Okita \\ Department of Cardiovascular Surgery, Kobe University, Kobe, Japan \\ Correspondence to: Yutaka Okita, MD. Professor, Division of Cardiovascular Surgery, Department of Surgery, Kobe University Graduate School of \\ Medicine, 7-5-2 Kusunoki-cho, Chuo-ku, Kobe, Japan. Email: yokita@med.kobe-u.ac.jp.
}

\begin{abstract}
Current surgical results of acute type A aortic dissection in Japan are presented. According to the annual survey by the Japanese Association of Thoracic Surgery, 4,444 patients with acute type A aortic dissection underwent surgical procedures and the overall hospital mortality was $9.1 \%$ in 2013 . The prevalence of aortic root replacement with a valve sparing technique, total arch replacement (TAR), and frozen stent graft are presented and strategies for thrombosed dissection or organ malperfusion syndrome secondary to acute aortic dissection are discussed.
\end{abstract}

Keywords: Acute aortic dissection; Japan; surgery

Submitted Jan 27, 2016. Accepted for publication Jun 10, 2016.

doi: 10.21037/acs.2016.06.02

View this article at: http://dx.doi.org/10.21037/acs.2016.06.02

\section{Introduction}

The acute aortic dissection is a dreadful disease that has a prevalence that has been increasing in number. This can be attributed to the development of an elderly society and also to physicians' awareness of aortic disease in the patients with chest pain. However, early mortality of surgery for acute aortic dissection has been around $10 \%$ even in experienced cardiac centers and there are still many patients dying before reaching the operating theatre.

The annual survey by the Japanese Association for Thoracic Surgery (JATS) covered surgical data from all of Japan, including early mortality. In 2013, 589 out of 602 $(97.8 \%)$ institutions participated in the survey (1). The annual number of operations for the thoracic aorta has steadily increased, from 712 in 1984 to 15,758 in 2013 (Figure 1). The number of surgeries for acute aortic dissection has increased, as well as of surgery for non-dissection (Figure 2). The outcomes of surgery have improved throughout. Hospital mortality after elective arch replacement improved to $7.2 \%$, and hospital mortality after emergent operation for acute type A aortic dissection was reported to be $9.1 \%$ in the JATS 2013 registry (Figures 3,4). Kazui et al. in 2007 (3) (Figure 5A) and Sakata et al. in 2012 (4) (Figure 5B) reported 5 -year analyses of the JATS survey data, and hospital volume (case load) and outcomes of surgery for the aortic arch or acute type A dissection were also correlated to some degree. They also underscored that there was a bi-phasic pattern of results, where even hospitals with a small case load could achieved good clinical outcomes.

The Japan Adult Cardiovascular Surgery Database (JCVSD) data includes all surgical procedures of the heart and of the thoracic aorta, because data collection has been a requirement for the Japanese Cardiovascular Surgery Board Certification since 2011. This JCVSD was characterized by collection of detailed data about surgery for the thoracic aorta (5) and the preoperative risk-stratified score (J score) can be calculated from the collected Japanese data.

\section{Aortic root replacement}

Aortic root replacement is indicated in patients with a destructive aortic root secondary to aortic dissection, such as intimal tear within the root, pre-existing annuloaortic ectasia or organic aortic valve lesions. The gold standard for replacing the aortic root has been the Bentall-DeBono operation since 1968, but complications associated with anticoagulation and structural or non-structural prosthesis deterioration have shifted the paradigm towards valvesparing aortic root operations, such as Yacoubructural prosthesis deterioration have shifted 1968, patients with acute aortic dissection usually have normal aortic cusps. 


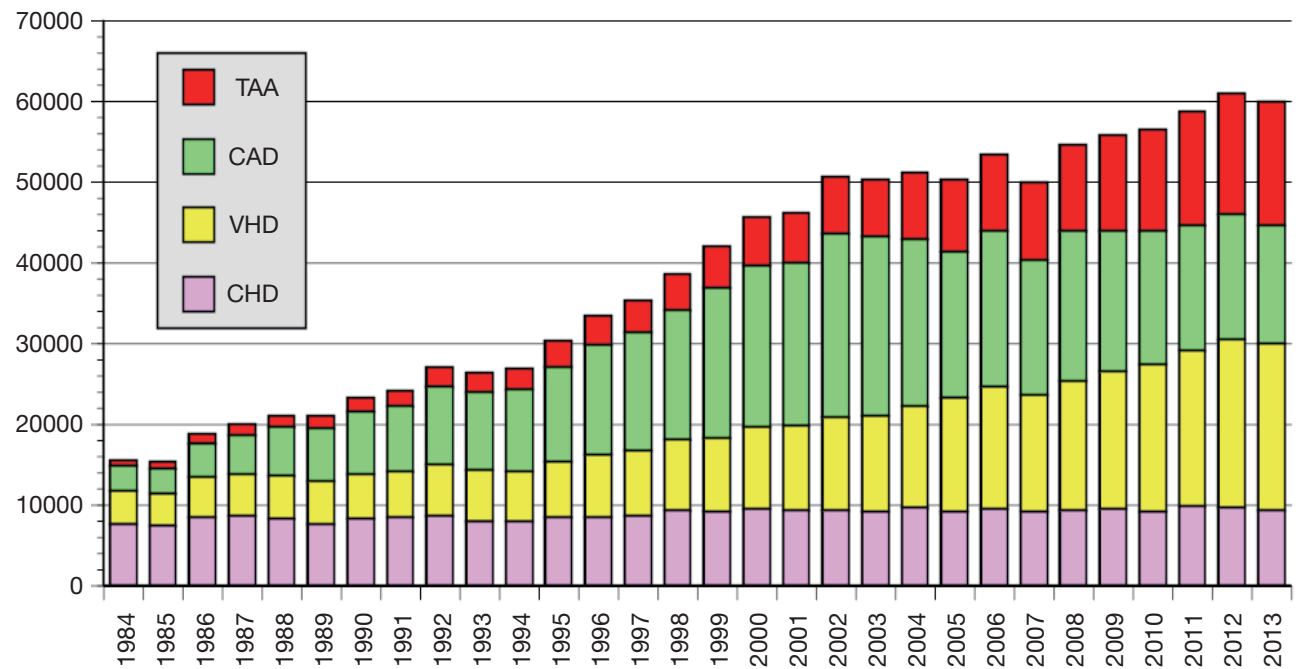

Figure 1 Annual Survey of Cardio-aortic Surgery [1984-2013] by Japanese Association for Thoracic Surgery. Modified from reference (2). TAA, thoracic aorta; CAD, coronary artery disease; VHD, valvular heart disease; CHD, congenital heart surgery.

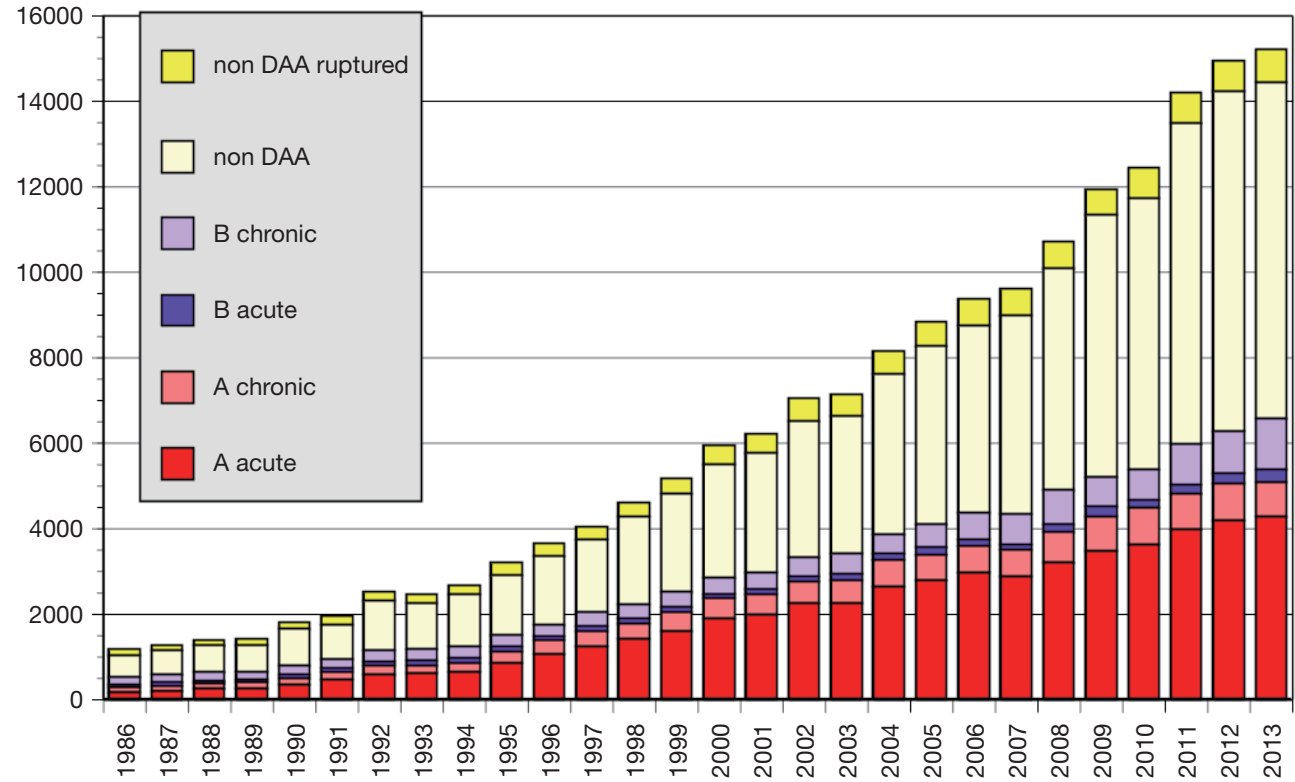

Figure 2 Annual Survey of Thoracic Aortic Surgery [1984-2013] by Japanese Association for Thoracic Surgery. Modified from reference (2). DAA, Aortic dissection; A Acute, acute type A aortic dissection; B Acute, acute type B aortic dissection; A Chronic, chronic type A aortic dissection; B Chronic, chronic type B aortic dissection; non DAA, non dissecting aneurysm; non DAA ruptured, ruptured aneurysm.

One of the advantages of aortic root replacement is complete elimination of the aortic lesion in the root, but this is counterbalanced by the complexity of the surgical procedures, especially by the valve sparing reimplantation technique. Serious complications of acute aortic dissection, such as aortic rupture, cardiac arrest, or major organ malperfusion, may be indicated to go for supra-coronary replacement of the ascending aorta, precluding valve sparing aortic root replacement.

In the recent debate over the superiority of the two procedures, no conclusions have been reached. As for patients with acute aortic dissection, the reimplantation 


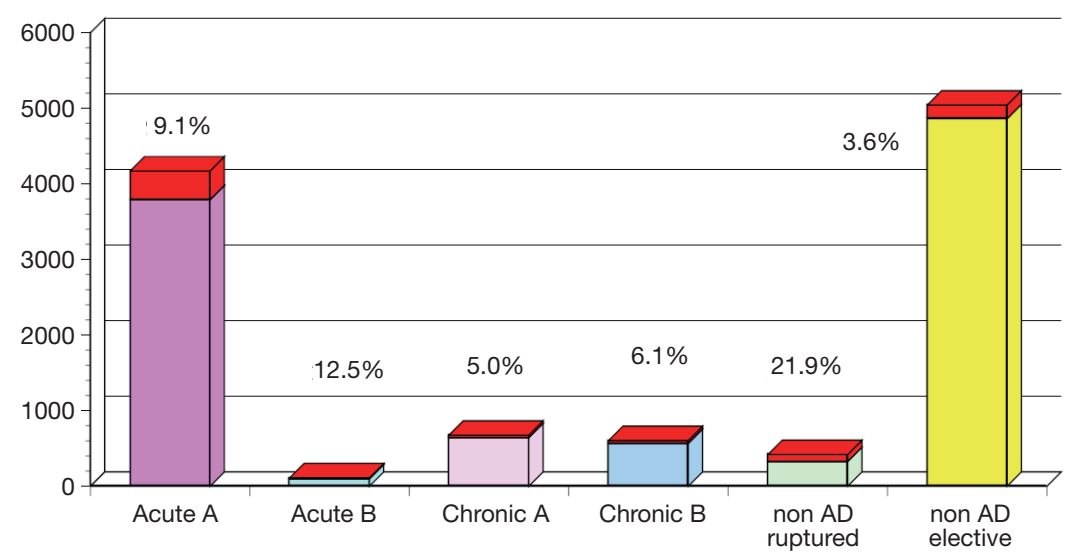

Figure 3 Hospital mortality in thoracic aortic surgery in 2013 ( $n=15,199)$. Japanese Association for Thoracic Surgery. Red bar represents hospital mortality. Modified from reference (2). AD, aortic dissection; Acute A, acute type A aortic dissection; Acute B, acute type B aortic dissection; Chronic A, chronic type A aortic dissection; Chronic B, chronic type B aortic dissection.
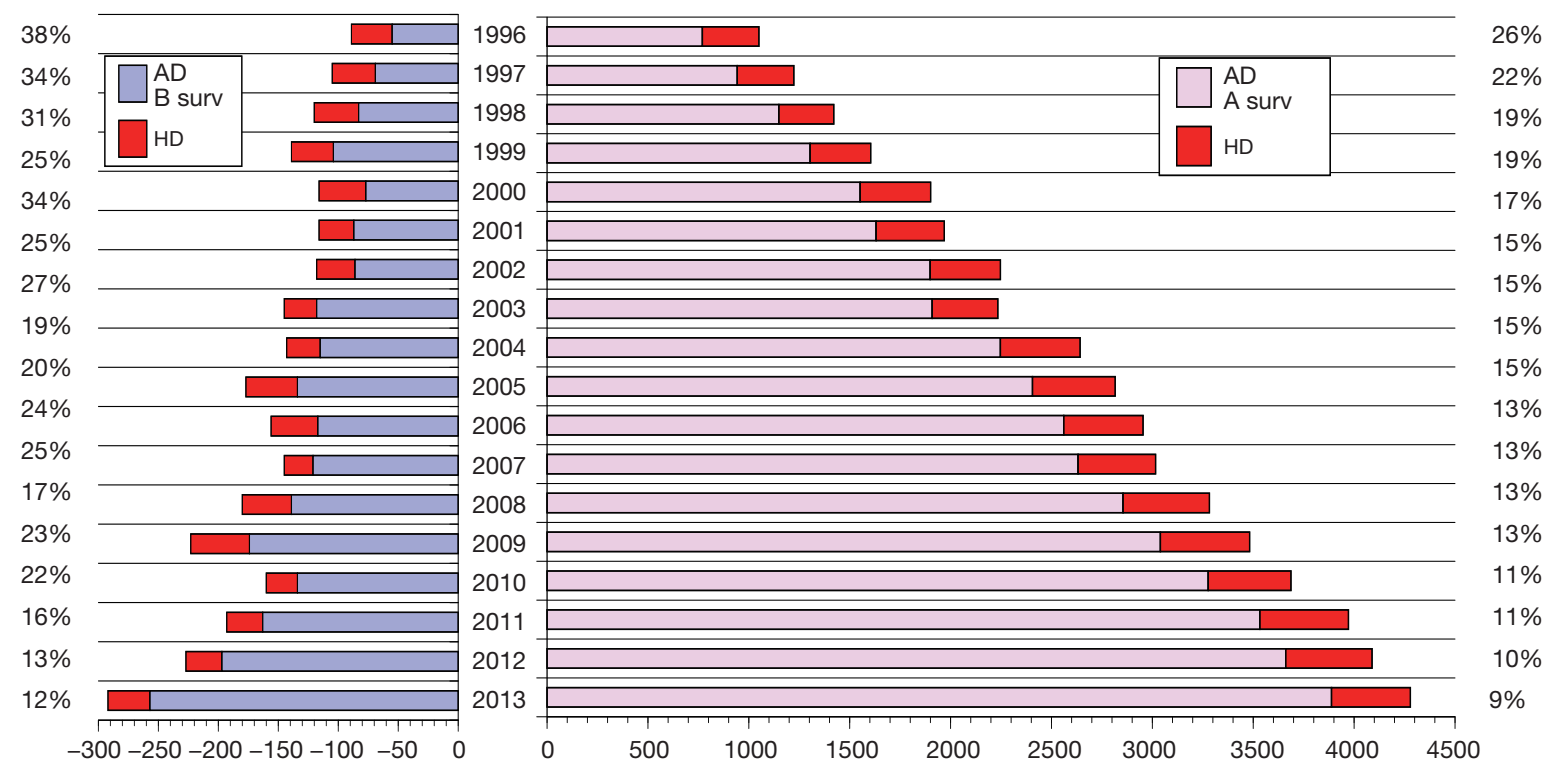

Figure 4 Acute aortic dissection \& hospital mortality. Japanese Association for Thoracic Surgery 1996-2013. Modified from reference (2). AD A surv, acute type A aortic dissection survival; AD B surv, acute type B aortic dissection survival; HD, hospital death.

technique provides more secure hemostasis and the remodeling technique is considered to be simpler. Not enough cases of the application of the aortic cusp-sparing root replacement in patients with acute aortic dissection have been accumulated. We performed aortic root reimplantation technique in 23 patients with acute aortic dissection and 11 had concomitant total arch replacement (TAR) in the Kobe University Hospital (6). Fortunately there was no early death, but five patients required redo
AVR because of detachment of the cusp commissures and tissue necrosis due to the biological glue.

\section{Non-communicating dissection}

There has been continuing discussion regarding the definition of thrombosed aortic dissection. In Western countries, the term intramural hematoma (IMH) is frequently used in guideline and clinical settings. They 

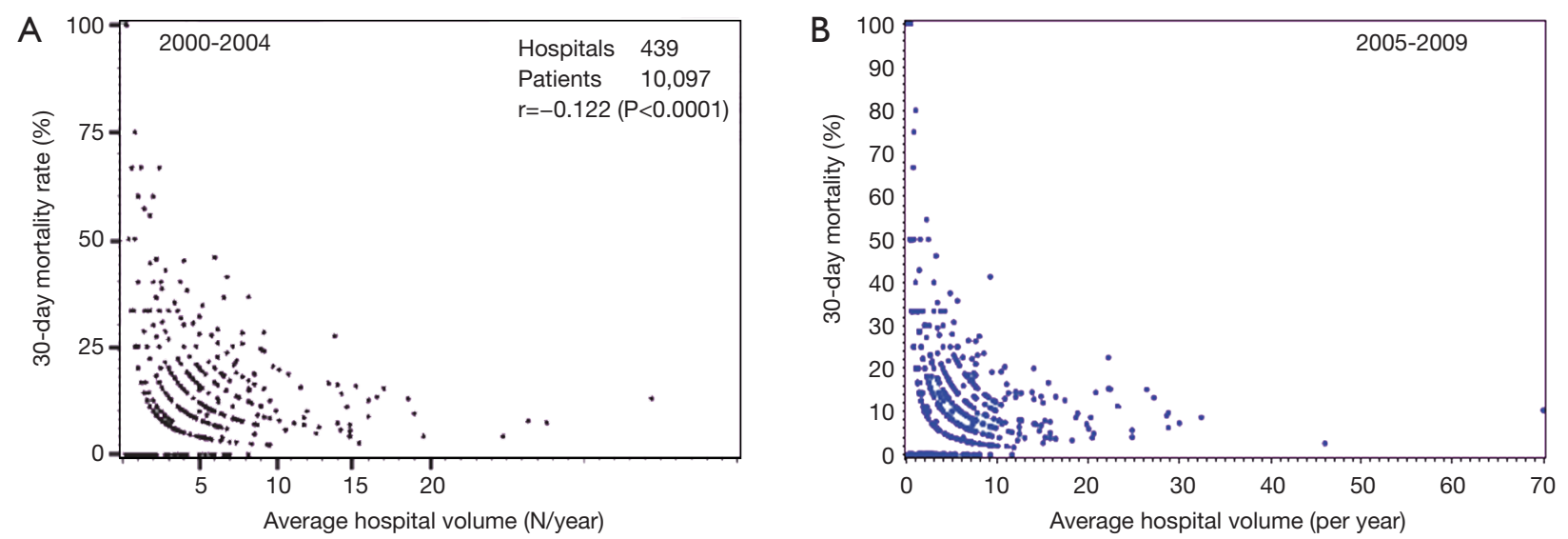

Figure 5 Early death related to case volume. (A) Relationship of 30-day mortality and case load of surgery for acute type A aortic dissection. 2000-2004. Japanese Association for Thoracic Surgery; (B) relationship of 30-day mortality and case load of surgery for acute type A aortic dissection. 2005-2009. Japanese Association for Thoracic Surgery. Quoted from reference (2).

sometimes included aortic dissection with a clear ulcerative projection (ULP) in the thrombosed false lumen as an IMH. As for thrombosed dissection, "partially thrombosed dissection" raises more ambiguity. In the Japanese Guideline for Thoracic Aortic Disease 2011, we defined the non-communicating aortic dissection as no communication between the false lumen and the true lumen (7). The ULP type dissection and non-communicating dissection are considered as different pathological conditions.

There is another discrepancy in the treatment strategy for type A non-communicating aortic dissection between Western countries and Japan or South Korea (7) as well as between surgeons and cardiologists within countries. Some surgeons recommend emergency surgery in all patients with non-communicating type A dissection (8), but we have set indications for emergency surgery in the patients who have complicated aortic valve insufficiency or cardiac tamponade, have a ULP in the ascending aorta, and patients with an aortic diameter of $50 \mathrm{~mm}$ or greater or a hematoma thickness of $11 \mathrm{~mm}$ or greater. In other cases without these findings, initial medical treatment seems possible. However, diagnostic imaging should be performed frequently during medical treatment, and it is advised that cases showing an increase in size of the thrombosed false lumen or a change to ULP or communicating type promptly undergo surgery.

\section{Arch replacement}

Principles of surgery for acute type A aortic dissection included artificial graft replacement of the aorta including entry. Since the majority of patients have an intimal tear in the ascending aorta, the standard technique has been ascending aortic replacement under an open distal anastomosis without aortic cross-clamping. The current standard brain protection is antegrade cerebral perfusion (ACP) with circulatory arrest of the lower body. Regarding the lowest body temperature during ACP, recent trends to avoid deep hypothermia have prevailed, but mild hypothermia is not safe for the brain, spinal cord, and visceral protection especially when the unilateral ACP is applied. Deep hypothermic circulatory arrest (HCA), by which the core temperature is reduced to $20^{\circ} \mathrm{C}$ or lower, has been the basic adjuvant procedure during arch reconstruction, while retrograde cerebral perfusion (RCP) may also be used concomitantly. Some reports stated that patients who had ACP had a lower incidence of transient neurological dysfunction (9). On the other hand, trying to minimize the duration of circulatory arrest of the brain with simple deep hypothermia or with RCP, the arch first technique had results that are similarly successful to those with ACP (10). Tokuda et al. (11) reviewed the results for two different methods: ACP and RCP, during ascending aortic repair for acute type A aortic dissection for the period between 2008 and 2012 using the Japan Adult Cardiovascular Surgery Database. They excluded patients who had root repair, arch vessel reconstruction and/or concomitant procedures. A total of 4,128 patients (ACP, $\mathrm{n}=2,769$; RCP, $\mathrm{n}=1,359$; mean age, $69.1 \pm 11.8$ years; male $41.9 \%)$ were identified. The overall operative mortality was $8.6 \%$. Between 1,320 propensity-matched pairs, cardiac 

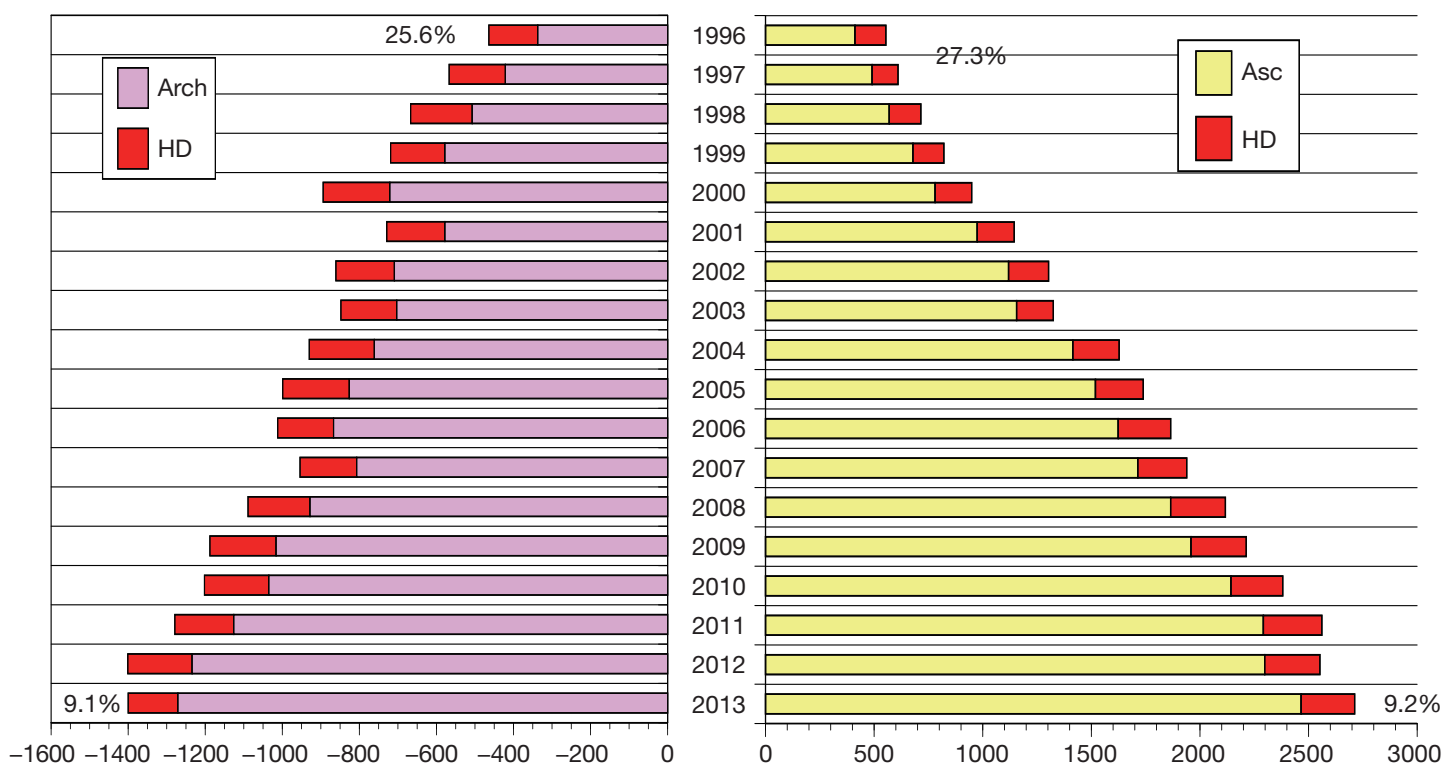

Figure 6 Hospital mortality of acute type A aortic dissection. Comparison of the method of surgery, ascending aorta or total arch replacement, Japanese Association for Thoracic Surgery survey. Modified from reference (2).

arrest time (ACP $116 \pm 36$ vs. RCP $102 \pm 38$ min, $\mathrm{P}<0.001$ ), perfusion time $(192 \pm 54$ vs. $174 \pm 53 \mathrm{~min}, \mathrm{P}<0.001)$ and operative time $(378 \pm 117$ vs. $340 \pm 108 \mathrm{~min}, \mathrm{P}<0.001)$ were significantly shorter in the RCP group. There were no significant differences between the two groups regarding the incidence of operative mortality or neurological complications, including stroke (ACP 11.2\% vs. RCP 9.7\%). Postoperative ventilation time was significantly longer in the ACP group (ACP 128.9 $\pm 355.7 v$ s. RCP $98.5 \pm 301.7$ hours, $\mathrm{P}=0.018$ ). There were no differences in other early postoperative complications, such as re-exploration, renal failure, and mediastinitis. They concluded that ACP is generally a safe method of brain protection but the most appropriate protective method may be chosen based on the individual case.

"Tear-oriented surgery" has been widely recommended during surgical repair of acute type A aortic dissection, and TAR is sometimes required. However, extensive graft replacement with TAR is more invasive and may carry an increased risk of morbidity and mortality. On the other hand, hemiarch replacement is the standard surgical approach for acute type A dissection; this limited graft replacement cannot excise an entry tear that is located in or beyond the aortic arch or sometimes complicated a newly developed intimal tear at the distal anastomotic line. Several studies have found that lack of an excised entry tear and a patent distal false lumen are risk factors for distal aorta expansion $(12,13)$. Dilatation of the distal false lumen during follow-up has driven surgeons to perform TAR in the acute type aortic dissection. TAR with installation of the elephant trunk into the true lumen of the descending aorta, wherever the entry site was, actually reduced the incidence of the late patency of the false lumen of the descending aorta and may have resulted in a decrease of the incidence of the re-operations $(12,13)$. It is very unique in the JATS surveys that one-third of patients who underwent surgery for acute type A aortic dissection had arch replacement. The 2013 Japanese survey revealed that hospital mortality after arch replacement for acute type A aortic dissection was found in $9.0 \%(151 / 1,504$ patients) compared to a rate of $10.0 \%$ $(252 / 2,805, \mathrm{P}>0.10)$ for ascending aorta repair (1) (Figure 6). This is an admirable achievement supported by recent refinement of the technique for ACP. Omura et al. (12) analyzed 197 patients with DeBakey type I acute dissection over 15 years and 109 had hemiarch and 88 had TAR. They reported that the frequency of distal aortic events, including redo for distal aorta enlargement or descending aorta more than $50 \mathrm{~mm}$, at 5 or 10 years was reduced in patients after TAR compared with ascending aorta replacement. Kimura et al. (13) reviewed 451 survivors after surgery for acute type A dissection over 23 years. Replacement of the whole aortic arch was done in only $66(14.4 \%)$ patients. They found that risk factors for late death were patent distal false lumen, older age, and male sex and risk factors for late aortic events 

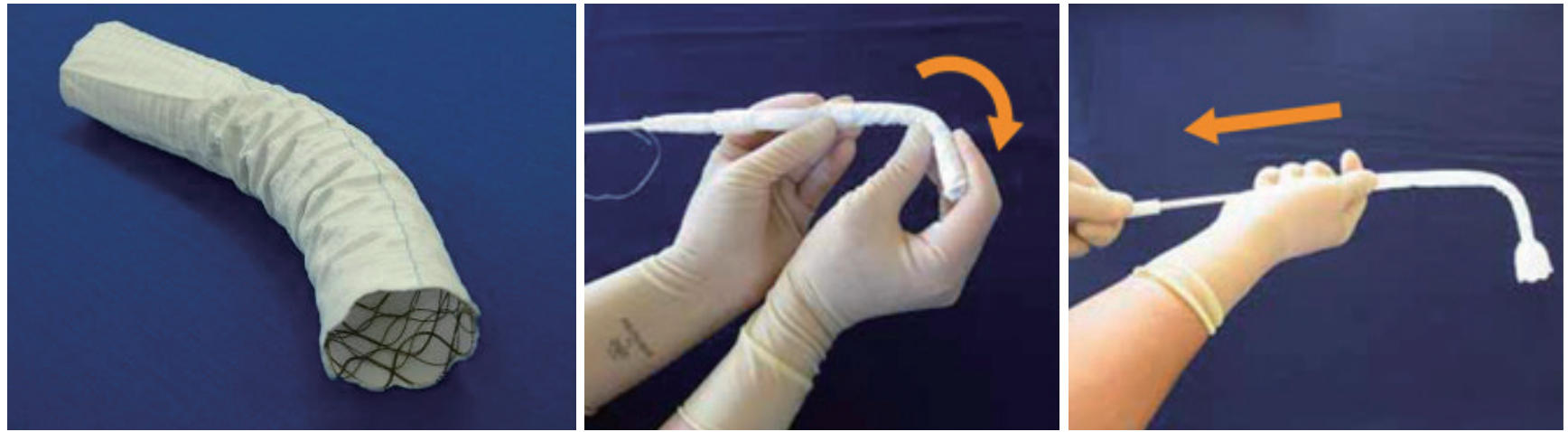

Figure 7 Open-stent graft (Frozenix ${ }^{\circledR}$, Japan Life Line Co.).

were patent distal false lumen, Marfan syndrome, and distal aorta over $45 \mathrm{~mm}$ in diameter. Fukui et al. (14) reported a superb early mortality of $5.2 \%$ in 504 patients with acute type A dissection over a 7-year period. The TAR was performed in 163 (32.3\%) patients.

The most important aspects of aortic arch surgery are considered to apply safe brain protection and to have secure anastomosis. The elephant trunk method has usually been used during TAR, with the aim of adding strength from the intimal side, preventing anastomosis leakage, and closing the peripheral false lumen. Open stent-grafting (OSG) or frozen elephant trunk (FET) (15) involves substituting distal anastomosis to the descending aortic suture by fixation with the stent-graft in aortic arch surgery. Simplifying the anastomosis of the descending aorta allows left thoracotomy to be avoided and reduces the time required for extracorporeal circulation. It has been reported that the outcomes in terms of the remaining false lumen are favorable when this technique is applied to aortic dissection (15). On the other hand, a number of reports have indicated that the incidence spinal cord injury is higher than with routine surgery $(15,16)$. The industry made open stent grafting (Frozenix ${ }^{\circledR}$, Japan Life Line, Tokyo, Figure 7), which was launched on the Japanese market in July 2014. So far, this OSG was used in 730 patients with acute type A dissection and 22 patients with type B dissection. A hybrid approach, combining OSG with the arch surgery, simplifies the strategy and has the potential to improve the late thrombosis of the false lumen of the descending aorta.

\section{Organ malperfusion: coronary, carotid, and superior mesenteric arteries}

Branch malperfusion is the main cause of increased complexity and severity of the disease state in cases with acute aortic dissection; this condition manifests with various symptoms in $20 \%$ to $40 \%$ of patients. The results of aortic dissection repair in patients who have a concomitant branch malperfusion are poor, and the early mortality rate is reported to be $30 \%$ to $50 \%$ (17).

The diagnosis of malperfusion should be started on suspicion of existence of malperfusion syndrome based on patients' signs and symptoms, such as consciousness disturbance, neurological deficits, or pain. Ultrasound is the mostly utilized clinical tools because malperfusion circulation changes over time, and ultrasound is the only objective tool that can instantaneously track the altered hemodynamics and perfusion status of the true or false lumen. Every patient should have echocardiography and carotid artery ultrasound simultaneously in the emergency room before going to the CT scan room. Orihashi (18) underscores the important role of the vascular ultrasound in the emergency room or operating room.

Multiple routes of blood perfusion should be used when there is pre-existing organ malperfusion. Organ malperfusion can occur even after cardiopulmonary bypass $(\mathrm{CPB})$ has started, particularly at the time of starting the bypass from the femoral arterial cannulation, or at onset of left ventricular fibrillation, or with aortic cross clamping. We always try to cannulate into the true lumen of the ascending aorta by the Seldinger method and closely monitor the blood pressure of both upper limbs and bilateral $\mathrm{rSO}_{2}$ on the foreheads to minimize this complication. When the malperfusion syndrome occurs after starting the CPB, the aortic clamp can be released if the aorta is not opened, or additional arterial cannulas are inserted in the axillary arteries or in the femoral arteries. Core cooling should be progressed and earlier installation 
of the ACP started (19).

In the Kobe University experience, arterial cannulation in two different sites was done in $17(5.2 \%)$ of 327 patients. Additional arterial cannulation was required in the axillary artery in 2 patients, in the ascending aorta in 2 , in the femoral artery in 1 , and in the left ventricular apex in 1 when arterial perfusion pressure or $\mathrm{rSO}_{2}$ dropped.

Patients with acute myocardial infarction in the left coronary area associated with acute type A dissection have rarely been saved unless the dissection itself was accidentally discovered during coronary angiography. Some report underscored the importance of preoperative PCI in such cases. Imoto et al. (20) reported 75 patients who had acute aortic dissection with coronary artery dissection, where involvement was in the right coronary artery (RCA) in 26 patients, the left coronary artery (LCA) in 19 and the RCA + LCA in 3. Preoperative coronary stent placement was performed in 7 patients (RCA, 4 and LCA, 3), aortic root replacement was done in 14 , coronary artery bypass grafting was done in 23 and biological glue was used in 28. Reported hospital death was $18 / 75$ patients (24\%) and they concluded that preoperative cardiopulmonary arrest (CPA) and myocardial ischemia (particularly LCA territory) negatively affect survival outcomes. Early revascularization by coronary stent placement is effective in preventing postoperative low cardiac out put syndrome.

New development of the neurological deficits is one of the highest risk factors for early death; patients with preoperative coma usually have tragic outcomes $(21,22)$. Some authors aggressively perfuse the carotid artery in the emergency ward (23), but some have proposed an intentional delay of surgery for such patients. We treated 43 patients with brain malperfusion among 312 patients $(13.8 \%)$ with acute aortic dissection. The preoperative consciousness level was coma in 7 patients, drowsy in 24 , and normal in 7. All underwent aortic repair and hospital mortality was $16.2 \%$ (7/43). Four had brain death secondary to huge hemispheric infarction. Five patients had temporary perfusion directly into the right common carotid artery and all survived with minimum postoperative neurological sequelae (23).

Similarly, patients who have the malperfusion of the abdominal viscera, especially in the superior mesenteric artery (SMA) region, deteriorate earlier and even emergent surgery for the proximal aorta cannot solve this problem. Recent advancement of the radiological intervention technique can sometimes revascularize the gut preoperatively and alleviate the metabolic crisis. Occasionally, preceding bypass surgery to the mesenteric artery is successful. Uchida et al. (24) reported successful outcomes of five patients who underwent preceding SMA revascularization and subsequent aortic repair. There has been controversy regarding the timing of the intervention for the central aorta and for the peripheral arteries. There has been consensus that if the aortic dissection shows an unstable course, aortic repair is the first priority, and intervention for peripheral vascular disease should be secondary (25). However, more aggressive reperfusion of the target organs, especially coronary arteries, carotid arteries, and SMA, before the aortic repair, may be recommended. In cases with unstable dissection such as ruptured aorta, cardiac tamponade, or severe AR, simultaneous repair of the central aorta and peripheral arteries should be prepared. When the malperfusion syndrome still persists after starting the $\mathrm{CPB}$, peripheral revascularization should be corrected. Otherwise irreversible ischemia of the end organ will ensue (17).

From October 2000 to December 2015, 327 consecutive patients with acute type A aortic dissection had surgery (mean age: $66.5 \pm 13.0,21-96$ ) in Kobe University hospital. Fifty-two patients were octogenarian or nonagenarian. All patients had surgery on an emergency or urgent basis. Seventy-three patients were in shock status and five required percutaneous assisted circulation. Salvage surgery after cardiopulmonary resuscitation was performed in 25 patients and 4 had "delayed surgery" because of serious preoperative stroke. There were 13 patients with Marfan syndrome and 14 patients had chronic kidney disease (serum creatinine $>2.0 \mathrm{mg} / \mathrm{dL}$ ). A total of 308 patients had an open false lumen, and 19 patients had non-communicating dissection. Cardiac tamponade was detected in 75 patients, and a moderate or severe aortic regurgitation in 53 patients, and 97 patients had some type of organ malperfusion syndrome (Figure 8). Fifty-one patients had neurological symptoms consisting of coma in 8 , stroke in 12 , TIA in 28 , and paraplegia in 3 . Fourteen patients had coronary malperfusion, 18 had visceral (SMA 9, renal artery 9) malperfusion and 33 had leg malperfusion. Twenty patients had 2 or more organ malperfusion. The entry sites were the aortic root in 6 , ascending aorta in 187 , aortic arc in 101 , proximal descending aorta in 23 , and undetected in 36. The extent of the aortic replacement was ascending aorta to hemiarch in 194, total arch in 133, and total arch to descending aorta in 2, and thoracic endovascular repair (TEVAR) in 1. Aortic valve resuspension or valve repair was performed in 298, root replacement with valve sparing in 21 and Bentall procedure in 8 . Brain protection was 


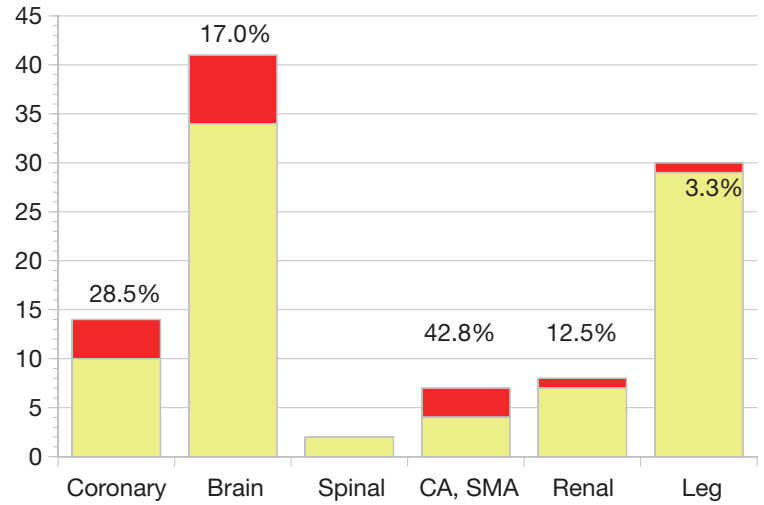

Figure 8 Incidence and early mortality of organ malperfusion syndrome complicated in acute type A aortic dissection. Kobe University 1999-2015/5 (n=97/308). Red bar represents hospital mortality. CA, celica artery; SMA, superior mesenteric artery.

achieved by DHCA with or without RCP in 79 , and ACP in 248. Additional procedures consisted with CABG in 7, and mitral valve repair in 2 . The overall hospital mortality was $13.7 \%$ [45] and the hospital mortality was $51.4 \%$ [18] in salvage surgery cases. Newly developed permanent neurological deficits occurred in 6.4\% [21] of patients and transient neurological disorder occurred in $6.1 \%$ [20]. There was no significant difference in the incidence of hospital death, stroke and TND between the DHCA and ACP group also between the patients who had hemiarch replacement and those who had TAR. Multivariate analysis demonstrated the risk factors for hospital mortality to be age (octogenarian; OR 1.05, $\mathrm{P}<0.001$ ), $\mathrm{CPB}$ time (OR $1.01, \mathrm{P}=0.0004)$, and salvage surgery (OR 10.8, $\mathrm{P}<0.001)$. Follow-up was completed in $97.2 \%$ of patients (mean $29 \pm 27,1-126$ months). Survival at 5 and 10 years after surgery was $75.8 \% \pm 2.7 \%$ and $71.7 \% \pm 3.3 \%$ respectively.

\section{Conclusions}

This report presents current surgical results of acute type A aortic dissection in Japan. According to the annual survey by the Japanese Association of Thoracic Surgery, 4,444 patients with acute type A aortic dissection underwent surgical procedures and the overall hospital mortality was $9.1 \%$ in 2013 . Aortic root replacement with a valve sparing technique in patients with acute aortic dissection is not widely performed in Japan. On the contrary, application of the TAR in acute aortic dissection has been more often performed in Japan. Recent launching of the frozen stent graft system in the Japanese market may accelerate this phenomenon. Aggressive surgery towards thrombosed aortic dissection provided excellent results. More efforts should be focused on establishing a surgical strategy for patients with acute aortic dissection complicated by organ malperfusion.

\section{Acknowledgements}

None.

\section{Footnote}

Conflicts of Interest: The author has no conflicts of interest to declare.

\section{References}

1. Committee for Scientific Affairs, The Japanese Association for Thoracic Surgery, Masuda M, et al. Thoracic and cardiovascular surgery in Japan during 2013: Annual report by The Japanese Association for Thoracic Surgery. Gen Thorac Cardiovasc Surg 2015;63:670-701.

2. Okita Y. Surgery for thoracic aortic disease in Japan: evolving strategies toward the growing enemies. Gen Thorac Cardiovasc Surg 2015;63:185-96.

3. Committee for Scientific Affairs, Kazui T, Osada H, et al. An attempt to analyze the relation between hospital surgical volume and clinical outcome. Gen Thorac Cardiovasc Surg 2007;55:483-92.

4. Sakata R, Kuwano H, Yokomise H. Hospital volume and outcomes of cardiothoracic surgery in Japan: 20052009 national survey. Gen Thorac Cardiovasc Surg 2012;60:625-38.

5. Motomura N, Miyata H, Tsukihara H, et al. Risk model of thoracic aortic surgery in 4707 cases from a nationwide single-race population through a web-based data entry system: the first report of 30-day and 30-day operative outcome risk models for thoracic aortic surgery. Circulation 2008;118:S153-9.

6. Minami H, Miyahara S, Okada K, et al. Clinical outcomes of combined aortic root reimplantation technique and total arch replacement. Eur J Cardiothorac Surg 2015;48:152-7.

7. JCS Joint Working Group. Guidelines for diagnosis and treatment of aortic aneurysm and aortic dissection (JCS 2011): digest version. Circ J 2013;77:789-828.

8. Shimokawa T, Ozawa N, Takanashi S, et al. Intermediateterm results of surgical treatment of acute intramural 
hematoma involving the ascending aorta. Ann Thorac Surg 2008;85:982-6.

9. Okita Y, Minatoya K, Tagusari O, et al. Prospective comparative study of brain protection in total aortic arch replacement: deep hypothermic circulatory arrest with retrograde cerebral perfusion or selective antegrade cerebral perfusion. Ann Thorac Surg 2001;72:72-9.

10. Okita Y, Miyata H, Motomura N, et al. A study of brain protection during total arch replacement comparing antegrade cerebral perfusion versus hypothermic circulatory arrest, with or without retrograde cerebral perfusion: analysis based on the Japan Adult Cardiovascular Surgery Database. J Thorac Cardiovasc Surg 2015;149:S65-73.

11. Tokuda Y, Miyata H, Motomura N, et al. Brain protection during ascending aortic repair for Stanford type A acute aortic dissection surgery. Nationwide analysis in Japan. Circ J 2014;78:2431-8.

12. Omura A, Miyahara S, Yamanaka K, et al. Early and late outcomes of repaired acute DeBakey type I aortic dissection after graft replacement. J Thorac Cardiovasc Surg 2016;151:341-8.

13. Kimura N, Itoh S, Yuri K, et al. Reoperation for enlargement of the distal aorta after initial surgery for acute type A aortic dissection. J Thorac Cardiovasc Surg 2015;149:S91-8.e1.

14. Fukui T, Tabata M, Morita S, et al. Gender differences in patients undergoing surgery for acute type A aortic dissection. J Thorac Cardiovasc Surg 2015;150:581-7.e1.

15. Kato M, Matsuda T, Kaneko M, et al. Outcomes of stent-graft treatment of false lumen in aortic dissection. Circulation 1998;98:II305-11; discussion II311-2.

16. Leontyev S, Tsagakis K, Pacini D, et al. Impact of clinical factors and surgical techniques on early outcome of patients treated with frozen elephant trunk technique by using EVITA open stent-graft: results of a multicentre study. Eur J Cardiothorac Surg 2016;49:660-6.

17. Okita Y, Takamoto S, Ando M, et al. Surgical strategies in managing organ malperfusion as a complication of aortic dissection. Eur J Cardiothorac Surg 1995;9:242-6; discussion 247.

18. Orihashi K. Mesenteric ischemia in acute aortic dissection. Surg Today 2016;46:509-16.

19. Shiiya N, Matsuzaki K, Kunihara T, et al. Management of vital organ malperfusion in acute aortic dissection: proposal of a mechanism-specific approach. Gen Thorac Cardiovasc Surg 2007;55:85-90.

20. Imoto K, Uchida K, Karube N, et al. Risk analysis and improvement of strategies in patients who have acute type A aortic dissection with coronary artery dissection. Eur J Cardiothorac Surg 2013;44:419-24; discussion 424-5.

21. Tanaka H, Okada K, Yamashita T, et al. Surgical results of acute aortic dissection complicated with cerebral malperfusion. Ann Thorac Surg 2005;80:72-6.

22. Morimoto N, Okada K, Okita Y. Lack of neurologic improvement after aortic repair for acute type A aortic dissection complicated by cerebral malperfusion: predictors and association with survival. J Thorac Cardiovasc Surg 2011;142:1540-4.

23. Okita Y, Matsumori M, Kano H. Direct reperfusion of the right common carotid artery prior to cardiopulmonary bypass in patients with brain malperfusion complicated with acute aortic dissection. Eur J Cardiothorac Surg 2016;49:1282-4.

24. Uchida K, Karube N, Yasuda S, et al. Pathophysiology and Surgical Treatment of Type A Acute Aortic Dissection. Jpn J Vasc Surg 2015;24:127-34.

25. Fann JI, Sarris GE, Mitchell RS, et al. Treatment of patients with aortic dissection presenting with peripheral vascular complications. Ann Surg 1990;212:705-13.
Cite this article as: Okita Y. Current surgical results of acute type A aortic dissection in Japan. Ann Cardiothorac Surg 2016;5(4):368-376. doi: 10.21037/acs.2016.06.02 